\title{
Determination of iron in solutions containing iron complexes
}

\author{
G. B. TENNANT AND D. A. GREENMAN \\ From the Department of Haematology, Welsh National School of Medicine, Cardiff
}

SYNOPSIS A method of estimating the iron content of solutions containing haemoglobin, ferritin, or ferrioxamine is described. Iron is released by treatment with acid permanganate and ascorbic acid before conventional determination by an AutoAnalyzer technique.

Methods in common use for the determination of complexed iron in compounds such as haemoglobin, ferritin, or ferrioxamine depend on a preliminary treatment to release free iron which is then estimated by a colorimetric reaction. The release of iron may be accomplished either by dry ashing in a furnace or wet ashing with hot concentrated acid (Bothwell and Finch, 1962). Both these procedures are timeconsuming and potentially hazardous. A simple technique is described for the release of iron from haemoglobin, ferritin, or ferrioxamine in solution by digestion with an acid-permanganate solution at room temperature. Excess permanganate is reduced with ascorbic acid, and iron in the resulting clear solution is estimated by the method of Young and Hicks (1965) using tripyridyl triazine.

\section{MATERIALS}

The following were used: potassium permanganate (Analar); hydrochloric acid (BDH volumetric solution); ascorbic acid (BDH biochemical reagent); concentrated sulphuric acid (BDH Aristar grade); desferrioxamine B mesylate (Desferal, Ciba); and ferritin $100 \mathrm{mg} / \mathrm{ml}$ aqueous solution (Koch-Light).

Iron standards containing $50,100,200,300,400$, and $500 \mu \mathrm{g}$ iron $/ 100 \mathrm{ml}$ were obtained by dilution with water from a stock solution of $20 \mathrm{mg} / 100 \mathrm{ml}$. This was prepared by dissolving $0.2 \mathrm{~g}$ pure iron wire in $100 \mathrm{ml}$ of $4 \mathrm{~N}$ nitric acid and diluting to 1 litre with water.

Deionized water was used throughout. All glassware was washed, acid-cleaned, and rinsed before drying.

\section{THE RELEASE OF IRON BY ACID-PERMANGANATE}

Fresh samples of venous blood taken into sequestrene were diluted 1 in 6 with water and estimations were carried out on the resultant haemolysates. To $0.6 \mathrm{ml}$ haemolysate was added $1 \mathrm{ml}$ of a mixture containing 4 parts potassium permanganate solution and 6 parts Received for publication 3 October 1968. hydrochloric acid in varying concentrations. This was mixed with a vortex mixer and left at room temperature. At the end of this digestion $0.4 \mathrm{ml}$ ascorbic acid solution of varying concentration was added and a sample transferred to a Technicon AutoAnalyzer for iron estimation by the method of Young and Hicks (1965).

POTASSIUM PERMANGANATE CONCENTRATION Complete release of iron from haemoglobin was obtained when $3 \%$ potassium permanganate solution was used (Fig. 1). This concentration was satisfactory for iron levels in original blood samples up to $80 \mathrm{mg}$ per $100 \mathrm{ml}(23 \mathrm{~g}$ haemoglobin per $100 \mathrm{ml}$ ).

HYDROCHLORIC ACID CONCENTRATION The use of very concentrated or very dilute acid resulted in the formation

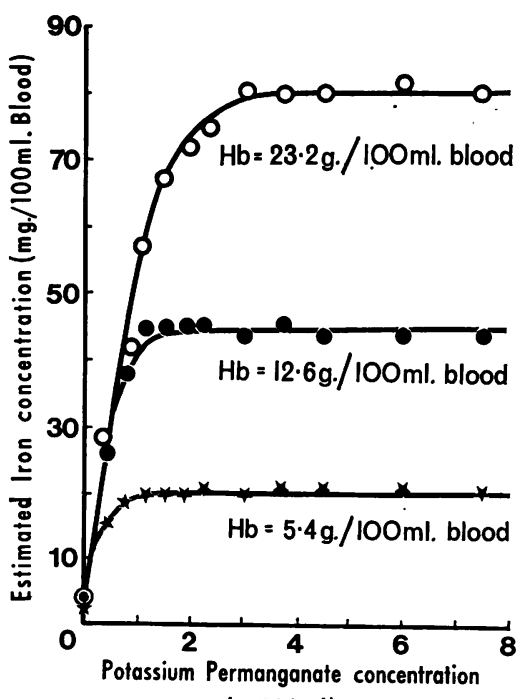

(g./100 $\mathrm{ml}$ )

FIG. 1. The effect of potassium permanganate concentration on the release of iron from haemoglobin. 


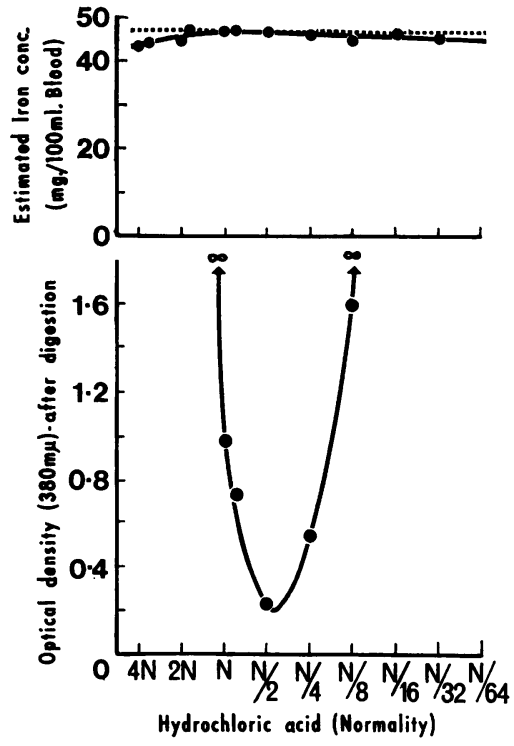

FIG. 2. Curves showing the effect of hydrochloric acid concentration on the optical density $(380 \mathrm{m \mu})$, and on the iron estimated in a haemoglobin digest $(\mathrm{Hb}=13 \cdot 6 \mathrm{~g} / 100 \mathrm{ml}$ $\equiv \mathrm{Fe}-47 \mathrm{mg} / 100 \mathrm{ml}$ ).

of a precipitate. This was minimal with $\mathrm{N} / 2$ acid and iron recovery using this concentration was $100 \%$ (Fig. 2). The optimum results were only obtained when the acid and permanganate were mixed before their addition to the haemolysate. Separate additions resulted either in precipitation or a dense yellow colour.

ASCORBIC ACID CONCENTRATION At low haemoglobin levels more ascorbic acid was needed to reduce the residual permanganate. An excess of ascorbic acid had no effect on the iron estimation and a $20 \%$ solution was used, which could fully reduce the $3 \%$ permanganate solution.

LENGTH OF DIGESTION Release of iron was complete after five minutes and extended digestion of up to six hours had no effect on the iron estimation. A 45-minute digestion was used in most of the present work but this period can be shortened.

TEMPERATURE OF DIGESTION The rate at which iron was released did not vary within the temperature range 4 to $56^{\circ} \mathrm{C}$.

DELAY BETWEEN ADDITION OF ASCORBIC ACID AND IRON ESTIMATION There was no change in the estimated level of iron in the solution with delays of up to several hours. Complete recovery was obtained when no delay occurred.

Solutions of ferritin and ferrioxamine released their iron in the same way as haemoglobin. The only exception was the behaviour of the ferritin digest after the addition of ascorbic acid. When the iron was estimated im- mediately, a very low yield was obtained but this increased progressively if the solution was left at room temperature and complete recovery only occurred after a 10-hour interval (Fig. 3), which could be considerably shortened by heating the mixture. The rate of increase in estimatable iron rose exponentially with temperature over the range $22-75^{\circ} \mathrm{C}$ and it was also directly related to the concentration of ferritin in the solution.

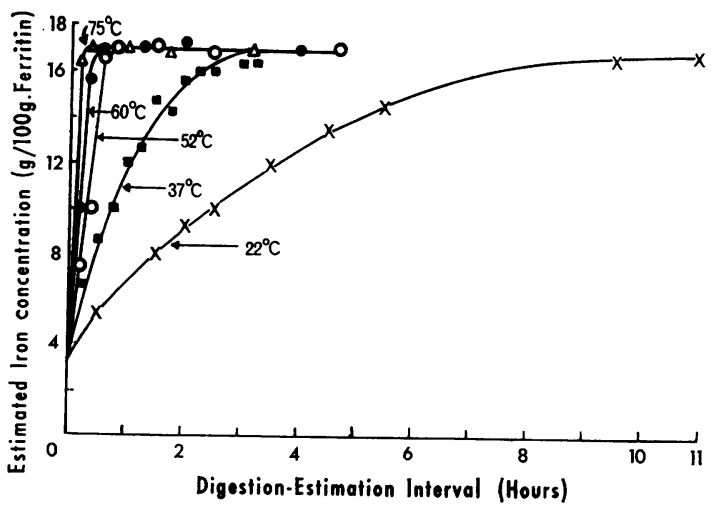

FIG. 3. The effect of delay between digestion and measurement on the estimation of iron released from ferritin. Acid-permanganate digestions were carried out on samples containing $1 \mathrm{mg}$ ferritin/ml at different temperatures.

\section{ROUTINE METHOD FOR IRON DETERMINATION}

1 Mix $0.6 \mathrm{ml}$ iron-containing solution and $1.0 \mathrm{ml}$ acid permanganate mixture $(4$ parts $3 \% \mathrm{w} / \mathrm{v}$ potassium permanganate and 6 parts $\mathrm{N} / 2$ hydrochloric acid).

2 Leave at room temperature for 15 minutes.

3 Add $0.4 \mathrm{ml} 20 \%$ ascorbic acid solution and mix.

4 If ferritin is likely to be present heat at $56^{\circ} \mathrm{C}$ for two hours.

5 Dilute as necessary and estimate iron using the method of Young and Hicks (1965) on the AutoAnalyzer.

\section{COMPARISON WITH STANDARD METHODS}

HAEMOGLOBIN The iron concentration of 40 samples of whole blood was measured by the present method. The calculated concentration of each sample was obtained from the measured haemoglobin concentration, using the cyanmethaemoglobin method and an ICSH reference standard, assuming the iron content of haemoglobin to be $0.347 \%$ (ICSH, 1967). The two values compared well (Fig. 4). The correlation coefficient was 0.99 and the regression coefficient 0.97 . The iron content of nine of the blood samples was also estimated by wet ashing with concentrated sulphuric acid followed by the standard AutoAnalyzer technique. This procedure gave results which agreed with those obtained by both the other methods. 


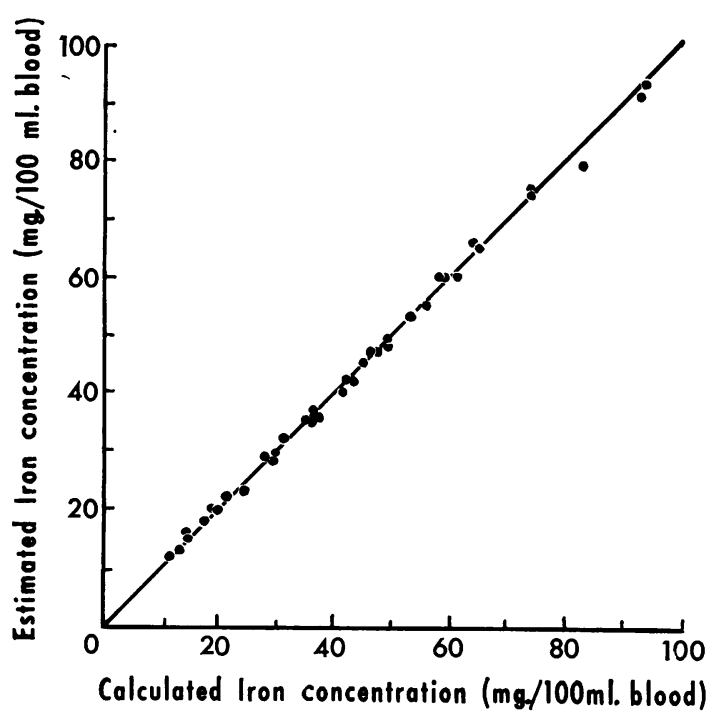

FIG. 4. Iron concentrations of $\mathbf{4 0}$ blood samples determined by the present method compared with those derived from haemoglobin estimations ( $1 \mathrm{~g} \mathrm{Hb}$ contains $3.47 \mathrm{mg} \mathrm{Fe}$ ).

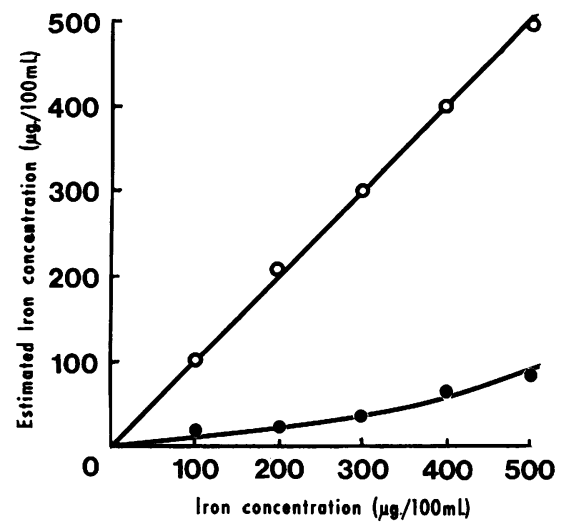

FIG. 5. Recovery of iron added to urine containing $10 \mathrm{mg}$ Desferal $/ 100 \mathrm{ml}$.

After acid-permanganate digestion with no treatment
FERRIOXAMINE Ferric iron was added to urine at concentrations of $100,200,300,400$, and $500 \mu \mathrm{g}$ per $100 \mathrm{ml}$, together with desferrioxamine B mesylate at a concentration of $10 \mathrm{mg}$ per $100 \mathrm{ml}$. Standard iron solutions were prepared from the stock solution by dilution in normal urine instead of in deionized water. Direct estimation of iron without prior permanganate treatment resulted in less than $20 \%$ recovery. One hundred per cent recovery was achieved using the present method (Fig. 5).

FERRITIN A $1 \mathrm{mg}$ per millilitre ferritin solution was prepared and this was used for a total of 14 iron estimations by the present method and nine estimations by wet ashing. The mean iron content of the ferritin by the wet ashing method was $16 \cdot 22 \pm$ $0.2 \%$ and by the permanganate method 16.89 $\pm 0 \cdot 15 \%$, ie, $104 \%$ recovery.

\section{CONCLUSION}

A method of estimating the iron content of solutions containing haemoglobin, ferritin, or ferrioxamine has been described which involves treatment with acid-permanganate before conventional determination by an AutoAnalyzer technique. It is rapid, robust, and convenient and gives satisfactory results. The presence of EDTA in the blood samples used did not interfere with the estimation of haemoglobin iron and an excess of desferrioxamine in urine did not interfere with the estimation of chelated iron. This method is probably suitable for the estimation of iron in many soluble biological complexes.

This work was partly supported by a grant from the Endowment Fund of the United Cardiff Hospitals to Dr A. Jacobs and we would like to thank him for his interest.

\section{REFERENCES}

Bothwell, T. H., and Finch, C. A. (1962). Iron Metabolism, p. 25 , Churchill, London. Little, Brown, Boston.

International Committee for Standardization in Haematology (1967). Brit. J. Haemat., 13, suppl., 71.

Young, D. S., and Hicks, J. M. (1965). J. clin. Path., 18, 98. 